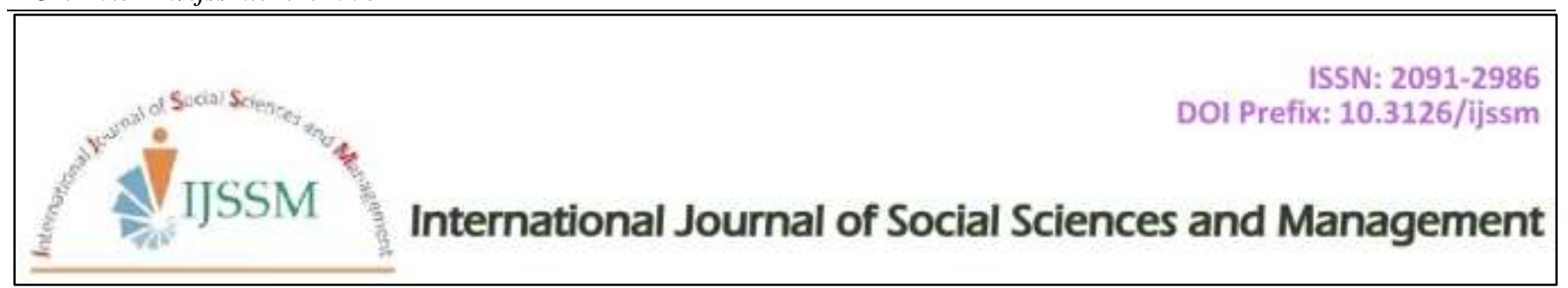

Mini Review

\title{
Monkeypox Transmission, Need and Treatment of Humans with an Antiviral Drug
}

\author{
Chanda Jabeen* and Gulshan Umbreen \\ Department of Epidemiology and Public Health, University of Veterinary and Animal Sciences Lahore, Pakistan \\ *Corresponding author's email: chandajabeen786@gmail.com
}

\begin{abstract}
Monkeypox is a viral zoonotic disease belongs to the family Poxviridae and Orthopoxvirus genus. Transmission of monkey pox is through direct contact with infected animal and blood. Human to human transmission occur through respiratory route but previously so many studies are conducted to prove that monkey pox viruse was not transmitted through the respiratory route both in animals and humans. But now monkey pox is able to survive in humans due to genetic changes and human to human transmission is possible. Because it can be used as bioweapon, So there is a great need of having an antiviral drug which is effective against monkey pox virus.ST 246 proved effective in vivo and in vitro in infected animals and trials done safely on non-infected humans but no data is available about the effectiveness of ST 246 on monkey pox or Orthopox infected human treated with ST 246.
\end{abstract}

Keywords: Monkeypox; Transmission; ST 246.

\section{Introduction}

Monkeypox is a viral zoonotic disease belongs to the family Poxviridae and Orthopoxvirus genus. Monkeypox was first discovered in a research lab in 1958 when two outbreaks of pock like disease occur in the monkeys which were kept their for research purpose, hence it is called monkey pox. Natural reservoir of monkey pox is still not known. Monkeypox has two genetic groups. Central African and West African. There is less number of death and human to human transmission in West African and vice versa in central African. Monkeypox is transmitted through direct contact with infected animal and blood and through respiratory droplets in human to human transmission (Nolen et al., 2016).

To identify the need of having an antiviral drug (ST-246) against monkey pox for treating infected humans.

\section{Inter Human Transmission}

Survey showed that $85 \%$ immunity against monkey pox comes from the small pox vaccination but human only caught infection by having contact with infected animals and there is no transmission of infection from human to human because viruse is not capable of surviving in human if vaccination is absent (Fine et al., 1988). Now monkey pox is able to survive in humans due to genetic changes (gene loss) and human to human transmission is possible. Virus is able to sustain in humans and spread can occur between humans due to genetic variation of the monkey poxvirus (Kugelman et al.,2014). WHO and health authorities observed $73 \%$ inter human transmission of monkeypox which was $28 \%$ according to the studies of WHO conducted in 1980 (Meyer et al.,2002). Monkey poxvirus spreads through inhalation when cynomolgus macaques inhaled the virus develop the disease and four out of six were killed humanly because of their serious condition. They inhaled $7.8 \times 10^{4} \mathrm{pfu}$ lethal dose (LD50) of monkey pox virus (Barnewall et al., 2012).

\section{Need for Having an Antiviral Drug ST-246}

A survey conducted in the demographic republic of Congo showed an increase number of people infected with monkey pox after stopping the small pox vaccine campaign (Rimoin et al., 2010). ST-246 proved an effective antiviral drug for the treatment of monkey pox in vitro by stopping the production of orthopoxvirus, and prevents the further release of viruse. No antiviral drug against the orthopox infection is approved by FAD ( Smith et al., 2009).

The smallpox vaccine ACAM 2000 efficiencies to cure monkeypox infection compared with anti-viral drug tecovirimat only and antiviral tecovirmat plus smallpox vaccine in a cynomolgusmacaque model after three days of 
getting infection. Then compared cynomolgusmacaque model with fatal monkeypox virus infection of third day exposure. No protection was provided by ACAM2000 against monkeypox disease when give alone after having infected with monkeypox virus and did not affect disease mortality. In comparison, tecovirimat provide full protection against monkeypox either given alone or in combination with small pox vaccine after having disease of monkeypox. Moreover, when given tecovirimat 4,5 , or 6 day after infection was effective $83 \%$ for days 4 and 5 and $50 \%$ effective for day 6 (Berhanu et al., 2015).

In a ground squirrel model of the disease efficiency of ST246 was evaluated for the treatment of monkey pox disease. Ground squirrels treated with $100 \mathrm{mg} / \mathrm{kg}$ of ST-246 per day orally after inoculating the lethal dose of MPX virus and then viruse inoculated and treatment given on first day and second day. Comparison done in placebo and control group for death, progression of disease, results of clinical laboratory and viral quantity. All animals survived lethal challenge with MPX virus that were given treatment of ST-246 after having exposure with infection on $0,1,2$ and $3^{\text {rd }}$ days; percentage of animals who were survived after having treatment with ST246 at $4^{\text {th }}$ post inoculation day of infection was $67 \%$. In contrast, percentage of death in placebo group is $100 \%$. The greater part of the ST-246-treated animals demonstrated no confirmation of clinical ailment or modification of pattern of clinical laboratories values and had insignificant histopathologic changes. These outcomes propose that ST246 is a potentially useful drug for treatment of serious orthopoxvirus disease (Sbrana et al., 2007).

Monkeypox infections, can bring about serious illness in people when conveyed by the airborne course, and consequently speak to huge dangers to both military and regular citizen populaces. As of now, there are no antiviral treatments affirmed by the U.S. Food and Drug Administration (FDA) to treat smallpox or monkeypox disease. Antiviral compound ST-246 administered to rabbits by oral gavage, once day by day for 14 days starting $1 \mathrm{~h}$ post exposure (p.e.), brought about $100 \%$ survival in a deadly aerosolized rabbit pox model utilized as a surrogate for smallpox. Moreover, adequacy of deferred treatment with ST-246 was assessed by starting treatment on days 1, 2, 3, and 4 p.e. In spite of the fact that a predetermined number of rabbits hinted at less extreme the rabbit pox ailment from the day 1 and day 2 p.e. treatment gathers, their ailment determined rapidly, and the survival rates for these gathering of rabbits were $88 \%$ and $100 \%$, separately. Be that as it may, when the treatment was begun on days 3 or 4 p.e., survival was $67 \%$ and $33 \%$, individually. This work proposes that ST246 is an exceptionally powerful antiviral compound against aerosolized rabbit pox in rabbits and ought to be explored for further advancement for all orthopoxvirus infections (Nalca et al., 2008).
ST-246, a strong orthopoxvirus departure inhibitor, is protected and powerful at averting infection and death in animal models including test by a few distinctive pathogenic pox viruses. The antiviral ampleness of ST-246 in treatment of nonhuman primates infected with variola disease or monkeypox was reviewed. The information show that oral dosing once every day with ST-246 shields animals from poxvirus disease, as measured by decreases in viral load and quantities of lesions and improvement of survival (Huggins et al., 2009).

\section{Treatment of Immune-Deficient Host with $S T$ - 246}

Danger of human monkeypox as an emerging disease and threat of smallpox as a bioweapon, among other pox viral diseases, highlight the requirement for compelling poxvirus countermeasures. ST-246, proved safe in human and give full safety against the development of deadly pox viral disease in numerous animal models by targeting the F13L protein in orthopoxvirus species. The efficacy of ST-246 is effective for immunocompetent animals as well as immunodeficient. ST-246 is effective in counteracting or treating deadly pox viral infection in immunodeficient mice. After deadly test with the Western Reserve strain of vaccinia, Nude, SCID, and JH knockout mice moreover drained of CD4+ and CD8+ T cells were not completely secured by ST246 , in spite of the fact that survival was essentially amplified. In any case, CD4+ T cell lacking, CD8+ T cell insufficient, $\mathrm{JH}$ knockout, and $\mathrm{JH}$ knockout mice likewise inadequate for CD4+ or CD8+ T cells survived deadly test when treated with ST-246 beginning upon the arrival of test. Postponing treatment until $72 \mathrm{~h}$ after disease diminished ST246 adequacy in a few models however gave full insurance from deadly test in most. These discoveries propose that ST246 may be successful in controlling smallpox or other pathogenic orthopoxviruses in some immunodeficient human populaces for whom the antibody is contraindicated (Grosenbach et al., 2010).

\section{Justification of Treatment of Human Monkeypox with Antiviral Drug ST-246}

Non-human primates were infected by introducing orthopox infection via intravenous route. These non-human primates were treated with ST 246 after third day of introducing virus. On 14 day at the dose of $10 \mathrm{mg} / \mathrm{kg}$ reduce the virus in blood which is equal to the $400 \mathrm{mg}$ in humans. So if $400 \mathrm{mg}$ of ST 246 given to the humans one time in a day it is effective against the monkeypox (Jordan et al., 2009).

ST 246 is safe for the human. ST 246 was given by mouth to the healthy individuals once time in a day and the doses were $500,1,000$, and 2,000 $\mathrm{mg}$ as a single dose all these individuals were having fast, and $1000 \mathrm{mg}$ given to the individuals who were not fasting. ST 246 was well tolerated in all these subjects and no complications found( Jordan et al., 2008). 
In mice and non-human primates preclinical safety pharmacology studies demonstrate that ST-246 is promptly consumed by the oral course and well tolerated with the no noticeable harmful impact level in mice when ST-246 given at $2000 \mathrm{mg} / \mathrm{kg}$ and there was no harmful impact level in nonhuman primates when $300 \mathrm{mg} / \mathrm{kg}$ of ST-246 given. ST-246 is protected and tolerated in healthy human volunteers demonstrated by human stage I clinical trials.

In light of the consequences of the clinical assessment, once every day dosing ought to give plasma drug level in the extent anticipated to be anti-viral taking into account based on data from efficacy studies in animal models of orthopoxvirus disease. These information sustain the utilization of ST-246 as an antiviral drug to treat pathogenic orthopoxvirus diseases of humans ( Jordan et al., 2010).

\section{Conclusion}

Formerly, Monkeypox was not transmitted through respiratory route and not able to sustain in humans but now it is possible due to genetic changes. So, there is a great need of having an antiviral drug for its treatment.

\section{Recommendation}

ST 246 proved effective in vivo and in vitro in infected animals and trials done safely on non- infected humans but no data is available about the effectiveness of ST 246 on monkeypox or orthopox infected human treated with ST 246. So, further studies should be done in this area.

\section{Acknowledgement}

Authors are thankful to Dr. Muhammad Hassan Mushtaq (Assistant Professor, Department of Epidemiology and Public Health, University of Veterinary and Animal Sciences, Lahore) for guidance with the topic of such great impact.

\section{References}

Barnewall RE, Fisher DA, Robertson AB, Vales PA, Knostman KA and Bigger JE (2012) Inhalational monkeypox virus infection in cynomolgus macaques. Frontiers in cellular and infection microbiology 2: 117.

Berhanu A, Prigge JT, Silvera PM, Honeychurch KM, Hruby DE and Grosenbach DW (2015) Treatment with Smallpox Antiviral Tecovirimat (ST-246®) alone or in Combination with ACAM2000® Vaccination is Effective as a PostSymptomatic Therapy for Monkeypox Virus Infection. Antimicrobial agents and chemotherapy 59(7): 4296-4300.

Fine P, Jezek Z, Grab B and Dixon H (1988)The transmission potential of monkeypox virus in human populations. International journal of epidemiology 17(3): 643-650.

Grosenbach DW, Berhanu A, King DS, Mosier S, Jones KF, Jordan RA, et al. (2010) Efficacy of ST-246 versus lethal poxvirus challenge in immunodeficient mice. Proceedings of the National Academy of Sciences 107(2): 838-843.
Huggins J, Goff A, Hensley L, Mucker E, Shamblin J, Wlazlowski C, et al. (2009) Nonhuman primates are protected from smallpox virus or monkeypox virus challenges by the antiviral drug ST-246. Antimicrobial agents and chemotherapy 53(6): 2620-2625.

Jordan R, Goff A, Frimm A, Corrado ML, Hensley LE, Byrd CM, et al. (2009) ST-246 antiviral efficacy in a nonhuman primate monkeypox model: determination of the minimal effective dose and human dose justification. Antimicrobial agents and chemotherapy 53(5): 1817-1822.

Jordan R, Leeds JM, Tyavanagimatt S and Hruby DE (2010) Development of ST-246® for treatment of poxvirus infections. Viruses 2(11): 2409-2435.

Jordan R, Tien D, Tove' CB, Jones KF, Tyavanagimatt SR, Strasser $\mathrm{J}$, et al. (2008) Single-dose safety and pharmacokinetics of ST-246, a novel orthopoxvirus egress inhibitor. Antimicrobial agents and chemotherapy 52(5): 1721-1727.

Kugelman JR, Johnston SC, Mulembakani PM, Kisalu N, Lee MS, Koroleva G, et al. (2014) Genomic variability of monkeypox virus among humans, Democratic Republic of the Congo. Emerging infectious diseases 20(2): 232.

Meyer H, Perrichot M, Stemmler M, Emmerich P, Schmitz H, Varaine F, et al. (2002) Outbreaks of disease suspected of being due to human monkeypox virus infection in the Democratic Republic of Congo in 2001. Journal of clinical microbiology 40(8): 2919-2921.

Nalca A, Hatkin JM, Garza NL, Nichols DK, Norris SW, Hruby DE, et al. (2008) Evaluation of orally delivered ST-246 as postexposure prophylactic and antiviral therapeutic in an aerosolized rabbitpox rabbit model. Antiviral research 79(2): 121-127.

Nolen LD, Osadebe L, Katomba J, Likofata J, Mukadi D, Monroe B , ... \& Bomponda PL (2016) Extended human-to-human transmission during a monkeypox outbreak in the Democratic Republic of the Congo. Emerging infectious diseases 22(6): 1014

Rimoin AW, Mulembakani PM, Johnston SC, Smith JOL, Kisalu NK, Kinkela TL, et al. (2010) Major increase in human monkeypox incidence 30 years after smallpox vaccination campaigns cease in the Democratic Republic of Congo. Proceedings of the National Academy of Sciences 107(37): 16262-16267.

Sbrana E, Jordan R, Hruby DE, Mateo RI, Xiao S-Y, Siirin M, et al. (2007) Efficacy of the antipoxvirus compound ST-246 for treatment of severe orthopoxvirus infection. The American journal of tropical medicine and hygiene 76(4): 768-773.

Smith SK, Olson VA, Karem KL, Jordan R, Hruby DE and Damon IK (2015) In vitro efficacy of ST246 against smallpox and monkeypox. Antimicrobial agents and chemotherapy 53(3): 1007-1012. 